Douglas R. Edwards, éd., Religion and Society in Roman Palestine. Old Questions, New Approaches

New York-Londres, Routletdge, 2004, 194 p.

URL : http://journals.openedition.org/assr/3511

DOI : 10.4000/assr.3511

ISSN : $1777-5825$

Éditeur

Éditions de l'EHESS

Édition imprimée

Date de publication : 1 mai 2006

Pagination : 147-299

ISBN : 2-7132-2092-0

ISSN : 0335-5985

Référence électronique

"Douglas R. Edwards, éd., Religion and Society in Roman Palestine. Old Questions, New Approaches ", Archives de sciences sociales des religions [En ligne], 134 | avril - juin 2006, document 134-31, mis en ligne le 18 octobre 2006, consulté le 21 septembre 2020. URL : http://journals.openedition.org/assr/ 3511 ; DOI : https://doi.org/10.4000/assr.3511

Ce document a été généré automatiquement le 21 septembre 2020.

(C) Archives de sciences sociales des religions 


\section{Douglas R. Edwards, éd., Religion and Society in Roman Palestine. Old Questions, New Approaches}

New York-Londres, Routletdge, 2004, 194 p.

1 «La conquête du Moyen-Orient, par Pompée en 63 av. J.-C., officialisa la chose. Rome allait contrôler la Palestine durant les 350 prochaines années. » C'est par cette entrée en matière que s'ouvre le livre dont l'objet est ainsi clairement annoncé : rendre compte, autant que faire se peut, à partir des données aujourd'hui disponibles, de la vie de la province romaine que fut la Palestine. L'intérêt de cet ouvrage est d'inviter les spécialistes de la culture matérielle à se confronter, une fois encore, aux problèmes et aux controverses soulevés par l'interprétation des découvertes archéologiques accumulées au cours des $\mathrm{XIX}^{\mathrm{e}}$ et $\mathrm{XX}^{\mathrm{e}}$ siècles.

2 C'est à cette confrontation que renvoient les "vieilles » questions et les «nouvelles " approches évoquées dans le sous-titre du livre. Pour en préciser les enjeux, on pourrait les rapporter à un problème: sur quelles bases scientifiques peut-on se fonder pour avancer des propositions un tant soit peu rigoureuses au sujet de l'interprétation des fouilles menées en Palestine tout en se départant des positions guidées par des inspirations nationales ou religieuses? En effet, si on parle de "nouvelles » approches appliquées aux résultats des fouilles, c'est surtout parce que la réflexion contemporaine s'oriente prioritairement sur les méthodes d'interprétation et cherche à dégager les précautions à prendre pour rendre compte de ces découvertes. Dans la présentation qu'il fait de cette entreprise, Martin Goodman, historien du judaïsme de la période romaine, rappelle que l'un des aspects les plus subtils des débats et questions soulevés depuis une cinquantaine d'années consiste à vouloir faire coïncider les recensions archéologiques avec la masse d'informations textuelles qui existent pour la période et qui, pour la plupart, sont des textes devenus quasiment canoniques pour les juifs et les chrétiens, sans pour autant être identiques. Le livre est dédié à Éric Meyer, dont la démarche a toujours été guidée par un principe : l'apparente contradiction entre les informations contenues dans les écrits et les éléments recueillis dans les fouilles archéologiques n'entamerait en rien la nécessité constante de référer les uns aux 
autres. De la même manière, le niveau local (épigraphique ou archéologique) devrait toujours être éclairé par la comparaison avec le niveau régional. C'est précisément cette approche qui est mise à l'épreuve dans ce livre. Qu'en résulte-t-il ?

3 La période la plus largement documentée par les sources littéraires est celle de l'occupation romaine de la Palestine, couverte par Flavius Josèphe au témoignage duquel on peut ajouter ceux de Philon d'Alexandrie et de Pline l'Ancien. C'est la période qui, autour du premier siècle, court en amont et en aval de la rédaction de la Bible hébraïque à celle du Nouveau Testament. Cette période est, précisément, celle qui a fait l'objet des débats les plus passionnés entre archéologues, dont la presse de vulgarisation a donné les plus larges relations en raison des hypothèses qui se sont affrontées, notamment à propos de celles concernant la paternité de la rédaction des "rouleaux de la mer Morte» et leur éventuel lien avec l'émergence du christianisme. Voilà qui donne toute sa pertinence à la confrontation des vestiges matériels archéologiques aux transmissions textuelles, soulignant ainsi l'intérêt de la démonstration méthodologique des contributions réunies dans l'ouvrage édité par D.R. Edwards.

4 L'historien, déjà coutumier de ce regard réflexif porté sur son travail, trouvera un intérêt à découvrir la façon dont les archéologues antiquisants chevronnés prennent ce souci en considération. Il notera ainsi que, bien que la démarche d'un archéologue à l'égard des fouilles ne soit pas identique à celle qu'il a, lui-même, à l'égard des textes, tous deux sont confrontés à l'obligation, en étudiant des sociétés disparues ou dont les témoignages sont épars ou inexistants, de reconstituer les blancs et les lacunes des sources documentaires. S'il peut paraitre simple de reconstituer la base d'une amphore ou de compléter les lettres manquantes d'un mot, ces reconstitutions s'avèreront plus ou moins heureuses selon que l'on aura ou non les moyens de les comparer avec d'autres objets ou lettres du même type pour en vérifier la vraisemblance ou la correspondance avec ce qui a existé.

5 Les treize contributions qui forment le livre vont du plus sobre compte rendu des résultats obtenus à ce jour par les archéologues aux débats plus âpres qui les divisent quant à l'interprétation du site de Qumran et à l'attribution des célèbres rouleaux trouvés sur le site ou dans sa proximité. L'espace manquant pour rendre compte en détail de chacune des treize contributions, on se contentera, pour donner une idée de l'importance de l'ensemble, d'évoquer les thèmes traités. Pour ouvrir le chantier, il convenait, sans doute, de reconstruire le monde de la Palestine romaine: exercice introductif auquel se livre l'éditeur de l'ouvrage, D.R. Edwards. Il fallait, ensuite, faire le point sur l'état actuel des recherches archéologiques sur le premier siècle. Mordechai Aviam dresse le tableau pour la Galilée, et Idan Shaked et Dina Avshalom-Gorni pour les implantations juives de la vallée située au sud-est du lac Hulé en discutant l'identification du site de Tel Talil au Thella mentionné par Flavius Josèphe. Milton Moreland s'interroge sur l'absence de réaction des Galiléens au christianisme primitif lors de sa rapide expansion en milieu urbain; tandis qu'Esther Eshel et D.R. Edwards tentent une analyse du degré de maîtrise de l'écriture et du langage des autochtones en se fondant sur un abécédaire en poterie découvert en Galilée. Puis c'est à la recherche de l'existence du culte de Dyonisos et d'Heraklès, à partir de la fresque en mosaïque exhumée à Séphoris, que nous invite Sean Freyne. Viennent ensuite une série de contributions destinées à présenter les points litigieux en cours: l'existence des synagogues au premier siècle, ainsi que les diverses hypothèses concernant l'origine de 
l'institution (sur un modèle égyptien ou gréco-romain), est analysée par Lee I. Levine (The Ancient Synagogues. The First Thousand Years, New Haven-Londres, Yale University Press, 2000), qui examine également les relations entre la synagogue du premier siècle et le Temple de Jérusalem, que les chercheurs ont souvent présentées comme conflictuelles. Levine saisit également cette occasion pour comparer les plans des synagogues de Palestine avec celles découvertes sur le site d'Ostie, et suggère qu'on peut en faire remonter les fondations à la fin du $\mathrm{I}^{\mathrm{er}}$ siècle. De façon plus fondamentale, il s'interroge sur la nature même de cette institution durant cette période : était-ce un centre communautaire par fonction (et donc profane) ou religieux par essence, comme il le fut plus tard au cours de l'Antiquité tardive, et si oui, dans quelle mesure?

6 L'analyse des pièces de monnaie, manne de l'archéologie, permet à Mark A. Chancey d'étudier l'exercice effectif du pouvoir romain de Pompée à la Grande Révolte en 135, en en étudiant les effigies qui marquent les avancées de la romanisation dans leur chronologie. Cynthia Baker part de l'imaginaire de la maison pour se livrer à une réflexion sur le sens à accorder aux notions de privé versus public. Renversant les $a$ priori courants sur la réclusion domestique des femmes, elle utilise les données fournies par les discussions rabbiniques pour établir qu'hommes et femmes étaient également à l'intérieur ou à l'extérieur d'espaces ; en effet, l'archéologie révèle que ces lieux étaient ouverts - sans murs ni cours closes. Elle en vient ensuite à discuter les attitudes de modestie prônées pour relever de la femme juive comme une rétro-projection des éléments culturels connus actuellement. Poussant plus avant sa réflexion sur la «maison », elle la compare à l'idée de nation qui regrouperait, en une sorte de grande famille, les fils et filles d'Israël, réhabilitée par le sionisme. La vie quotidienne et les questions de genre sont examinées par Myriam Peskowitz dans une étude sur le tissage et ses instruments. Son texte est, incidemment, une heureuse démonstration de la manière dont une chercheuse peut procéder lorsqu'il ne reste aucune trace tangible des objets dont elle rend compte mais qu'elle dispose de descriptions faites par ailleurs.

7 Viennent enfin les trois derniers articles consacrés au site de Qumran et aux rouleaux et qui constituent peut-être l'arrière-plan du volume. «Pourquoi des (rouleaux dans des) jarres? " se demande Jodi Magness, tout en répondant à l'aide des fragments QQMMT (miqetsat ma'asseh Torah) et 4Q274 3 II (4Q Tohorot A) : parce que les Esséniens devaient y déposer, afin de les protéger, leur nourriture et les écrits saints. Magen Broshi et Hanan Eshel affirment d'entrée que leur interprétation du site «est largement partagée par les spécialistes du domaine et est la seule qui soit correcte; Qumran était un monastère essénien et les rouleaux proviennent d'une communauté essénienne" (p.162). Ils présentent les raisons pour lesquelles les douze autres théories, avancées par d'autres archéologues et historiens quant à l'interprétation du site et de ses rouleaux, ne sont pas pertinentes. Pour finir, Jürgen Zangenberg offre un point de vue et une perspective plus nuancés en rassemblant les similitudes retrouvées entre le site de Khirbet Qumran et les autres villes de la région, élément qui ruine à lui seul la théorie de l'unicité du site et de l'isolement de ses habitants sur laquelle repose, radicalement, la thèse selon laquelle le site était un monastère.

8 Pour se faire une idée plus précise de la méthode utilisée par les archéologues réunis dans ce collectif, on peut prendre pour exemple la contribution de M. Aviam qui présente un état des lieux précis concernant les constructions et bâtiments, les objets et les ustensiles exhumés, rapportés au $\mathrm{I}^{\mathrm{er}}$ siècle. La période circonscrite va de la révolution hasmonéenne (fin du II ${ }^{\mathrm{e}}$ siècle av. J.-C.), qu'accompagnent les conquêtes de la 
région, jusqu'au règne d'Hérode Antipas à la fin du $\mathrm{I}^{\mathrm{er}}$ siècle. La conquête se manifeste par l'accumulation de céramiques grossières (remplacées au cours de la période juive par des vaisselles de pierre) sur de nombreux sites montagneux galiléens qui témoignent - comme celui de Mitspé ha-yamim - qu'un événement violent mit à bas les statues et conduisit à l'abandon de ce qui semble avoir été un temple ou un lieu de culte païen. Si en ce cas de figure, sources textuelles et vestiges matériels peuvent coïncider, il n'en va pas de même lorsqu'il s'agit de comparer l'allure générale des villes galiléennes aux descriptions effectuées par Flavius Josèphe. En effet, alors que celui-ci affirme que, dès avant l'entreprise de fortification, nombre de villes - comme Tibériade, Séphoris, Jafa (Yafi'a) ou Giscala (actuellement Gush Halav) - étaient ceintes de murs, les archéologues n'ont trouvé aucune trace de telles fondations, tandis qu'ils ont mis au jour celles de Gamla, Yodefat, Beer Sheva (en Galilée), du mont Thabor et d'Arbel. De l'assemblage des divers éléments exhumés, Aviam conclut que la Galilée s'est surtout développée en centre d'autonomie économique, vecteur de multiples et fréquents échanges, avec Jérusalem et les autres villes de la région, au cours de la période, relativement calme, d'Hérode Antipas.

9 Le lecteur non-initié aux comptes rendus archéologiques spécialisés pourrait être quelque peu dérouté par certaines de ces contributions, mais rien n'assure qu'il ne soit pas moins troublé par certaines autres, ne présentant pas le caractère spécialisé d'une étude d'éléments matériels et débattant des hypothèses pointues que leur interprétation suscite. Pourtant, on doit recommander à ce lecteur néophyte de dépasser ces détails techniques et de se laisser guider par les thèmes généraux discutés. Il découvrira, alors, que les questions toujours ouvertes en archéologie ressemblent à celles que les historiens "classiques" posent aux sources historiques à propos des juifs : quel fut leur niveau d'acculturation? À quel degré d'intégration parvinrent-ils dans les civilisations ? Comment maintinrent-ils leurs différences et établirent-ils leur sens de la singularité dans des sociétés multiculturelles (p.xv) ? Il y a gros à parier que le lecteur trouvera dans cet ouvrage ample matière à réflexion et nombre d'informations utiles. 